\title{
BMJ Open Risk factors for contrast-induced acute kidney injury (CI-AKI): protocol for systematic review and meta-analysis
}

\author{
Yong Liu, ${ }^{1}$ Xingcheng Liang, ${ }^{1,2}$ Shaojun Xin, ${ }^{1,2}$ Jin Liu, ${ }^{1}$ Guoli Sun, ${ }^{1}$ Shiqun Chen, ${ }^{1}$ \\ Xiaolin Cen, ${ }^{1,2}$ Xiaohua Dai, ${ }^{1,2}$ Yibo He, ${ }^{1}$ Feier Song, ${ }^{1}$ Yan Liang, ${ }^{3}$ Yuying Hu, ${ }^{4}$ \\ Yingling Zhou, ${ }^{1}$ Zhujun Chen, ${ }^{1}$ Ning Tan, ${ }^{1}$ Jiyan Chen ${ }^{1}$
}

To cite: Liu Y, Liang $X$, Xin S, et al. Risk factors for contrast-induced acute kidney injury (Cl-AKI): protocol for systematic review and meta-analysis. BMJ Open 2019:9:e030048. doi:10.1136/ bmjopen-2019-030048

- Prepublication history and additional material for this paper are available online. To view please visit the journal (http:// dx.doi.org/10.1136/bmjopen2019-030048).

$\mathrm{YL}, \mathrm{XL}, \mathrm{SX}, \mathrm{JL}$ and GS are joint first authors.

Received 27 February 2019

Revised 04 July 2019

Accepted 09 July 2019

\section{ABSTRACT}

Introduction Identifying the patients who are at risk for contrast-induced acute kidney injury (Cl-AKI), which is defined as an increase in serum creatinine after exposure to contrast media, is a critical step in targeted prevention strategies. The absolute and relative importance of individual risk factors have not been systematically evaluated, let alone the new, controversial and modifiable risk factors of $\mathrm{Cl}-\mathrm{AKI}$

Methods and analysis On 1 July 2019, a search was performed on MEDLINE, Embase and the Cochrane Database of Systematic Reviews. We will perform a systematic review and meta-analysis to assess the important risk factors for developing $\mathrm{Cl}$-AKI, including those new, modifiable factors, which are considered controversial. The secondary endpoint will be all-cause mortality. Two authors will then independently screen studies that meet the criteria for inclusion, consulting with a third author to resolve any dispute. The quality of the included studies will be assessed according to the Newcastle-Ottawa scale.

Ethics and dissemination Ethics approval in this systematic review and meta-analysis protocol is not needed. We will disseminate the findings of this systematic review and meta-analysis via publications in peerreviewed journals.

PROSPERO registration number CRD42019121534

\section{INTRODUCTION}

Contrast-induced acute kidney injury (CI-AKI) has been reported to be associated with the poor clinical outcomes including increased short-term and long-term mortality, prolonged duration of hospital stay, the need for renal replacement therapy and an increase in major adverse cardiac events. ${ }^{12}$ The use of a risk prediction tool for CI-AKI could have several benefits. Primarily, it may help to identify those patients at high risk for the disorder who might benefit from some prevention strategies like intravenous isotonic saline hydration that has been proven to be effective, statins and acetylcysteine that are controversial, or other interventions targeting the risk factors that will be identified in this meta-analysis in progress. ${ }^{3}$

\section{Strengths and limitations of this study}

- This will be the first, and largest, comprehensive systematic review of the diverse risk factors associated with contrast-induced acute kidney injury (Cl-AKI).

- Our broad search strategy will identify the important risk factors for $\mathrm{Cl}-\mathrm{AKI}$ for the purpose of preferentially supplying target prevention strategies, bringing attention to infrequently reported risk factors, and identifying new strategies to predict the risk of Cl-AKI.

- This study will also evaluate the new, modifiable risk factors, which are still considered controversial but may hold promise as preventive targets in the future.

- This study will only select English-language articles from the peer-reviewed literature.

Critical predisposing factors for CI-AKI include older age, pre-existing renal failure, haemodynamic instability, congestive heart failure, diabetes mellitus, anaemia and the volume of contrast media. ${ }^{45}$ Although Allen et at and Silver $e t a l^{7}$ systematically evaluated the current predictive models for CI-AKI, there has been no systematic assessment of the absolute and relative importance of the individual risk factors for CI-AKI, let alone, the new, modifiable and controversial one, for example, the usage of ACEI/ARB or diuretics, smoking and body mass index. ${ }^{3}$ This will be the first and largest systematic review about the risk factors associated with CI-AKI. To address this deficit in knowledge, we will conduct a systematic review and a meta-analysis of the observational studies that have examined the absolute and relative importance of the risk factors of CI-AKI.

\section{OBJECTIVES}

The objective of this study is to identity the relationship between the multiple risk factors of CI-AKI. More specifically, the goals of this study are the following: 
1. To comprehensively and systematically assess the absolute and relative importance of the current common risk factors for CI-AKI.

2. To systematically assess the new, controversial risk factors for CI-AKI.

\section{METHOD}

\section{Patient and public involvement}

This is a protocol for a meta-analysis, and it will be not appropriate or possible to involve patients or the public in this work.

\section{Search strategy}

A systematic computer-aided search of related studies will be conducted in the following databases:

1. Ovid MEDLINE (1946 to 30 June 2019, including epub ahead of print, in process and other non-indexed citations and daily).

2. Ovid Embase (1947 to June 2019).

3. Cochrane Database of Systematic Reviews (published on or before 30th June 2019).

\section{Initial keywords}

The databases will be searched using the following initial keyword search terms: coronary angiography, angiocardiograph, contrast medium, radiocontrast medium, AKI, contrast-induced nephropathy, acute renal insufficiency, risk factor, risk assessment, multivariate analysis and multivariable logistic regression; models. We will restrict the search on the databases to English language publications. If we find additional relevant keywords during any of the electronic searches, we will update the electronic search strategies with these terms and document the changes (details in online supplementary file).

\section{Types of studies}

We will include all observational studies (registries, cohorts, etc) that analyse the correlation between risk factors and CI-AKI following procedures with contrast media. This review will also consider experimental and epidemiological study designs, including case-control and cohort studies, as being appropriate for inclusion in the meta-analysis. However, we will exclude articles related to animal experiments.

\section{Types of participants}

We will include adults identified with the CI-AKI who are aged 18 years and above.

\section{Types of outcomes}

\section{Primary outcome}

The primary endpoint will be CI-AKI, defined as an increase in serum creatinine after exposure to contrast media.

\section{Secondary outcome}

The secondary endpoint will be long-term all-cause mortality, defined as death from any cause within the follow-up time of patient's postindex procedure.

\section{Selection of studies}

We will filter all retrieved articles. Two authors (XL and SX) will browse the titles and abstracts independently, and will record the excluded documents and the reasons for the exclusion. If there is a dispute in the process of the screening of the article, there will be a third author (YL) to arbitrate. After the initial screening, the two authors (JL and GS) will then read the full text and further filter the articles according to the inclusion and exclusion criteria mentioned above. To avoid overlapping patient data in duplicate publications, registry analyses will be crosschecked with institutional studies and compared with other registry studies, and the larger or more complete publication will be included. In the process of reading the full text, if there is doubt in the selection, the two authors (XL and SX) will discuss it. If it cannot be resolved, there will be a third author (YL) to arbitrate. Finally, all selected articles will be listed for further analysis, and the excluded articles will be classified according to the reasons for exclusion.

\section{Quality assessment/assessment of the risk of bias in included studies}

Two authors will independently assess the quality of selected articles using the Newcastle-Ottawa Scale. ${ }^{8}$

Observational studies using Newcastle-0ttawa Scale

Case-control studies

Selection

1 . Is the case definition adequate?

2. Representativeness of the cases.

3. Selection of controls.

4. Definition of controls.

Comparability

1. Comparability of cases and controls on the basis of the design or analysis.

Exposure

1. Ascertainment of exposure.

2. Non-response rate.

\section{Cohort studies}

Selection

1. Representativeness of the exposed cohort.

2. Selection of the non-exposed cohort.

3. Ascertainment of exposure.

4. Demonstration that outcome of interest was not present at start of study.

Comparability

1. Comparability of cohorts on the basis of the design or analysis.

Outcome

1. Assessment of outcome.

2. Was follow-up long enough for outcomes to occur.

3. Adequacy of follow-up of cohorts.

We will rate each selected article with a rating of low, high or unclear, and the results will be presented in the form of a table. In addition, we will use the Risk of Bias in Non-randomized Studies of Interventions Tool $^{9}$ to enhance the 
reproducibility and comparability of this review to future reviews of a similar topic.

\section{Data synthesis}

We will use STATA V.13.0 software and RevMan for data analysis, and risk factor effect sizes will be expressed as an OR and their $95 \% \mathrm{Cl}$. Using standard $\mathrm{X}^{2}$ test to assess the heterogeneity and variable statistics, we will perform subgroup analysis to conduct data statistics. If there are data that cannot be counted in the selected article, the data will be listed in the form of a table or chart. EndNote will be used for managing references.

Sensitivity analysis. We will perform sensitivity analyses in order to explore the influence of the following factors on effect sizes:

1. Restricting the analysis to studies of coronary angiography.

2. Restricting the analysis to large cohort studies with more than 1000 subjects.

\section{Meta-biases}

We will evaluate the possibility of publication bias using funnel plots and take Egger's test of bias as a complement. Unpublished data will also be considered in the evaluation of the publication bias, and we will try to contact the author for assistance in this regard.

\section{Confidence in cumulative evidence}

We will evaluate the strength of evidence for all outcomes by performing the Grading of Recommendations Assessment, Development and Evaluation working group methodology. The quality of evidence will be assessed across the domains of risk of bias, precision, directness, consistency and publication bias. Strength will be judged as high (further research is improbable to alter our confidence in the estimate of effect), moderate (further research will probably generate an important impact on our confidence in the estimate of effect and may alter the estimate), low (further research is very likely to generate an important impact on our confidence in the estimate of effect and change the estimate) or very low (the estimate of effect is indeterminable). ${ }^{10}$

\section{CONCLUSION}

The goal of this systematic review and meta-analysis is to clearly identify the important risk factors for CI-AKI for the purpose of preferentially supplying target prevention strategies, including contrast limiting and hydration for those high-risk factors. In addition, the modifiable, new and controversial factors will also be evaluated, as they may have potential as preventive targets.

\section{Author affiliations}

${ }^{1}$ Department of Cardiology, Guangdong Provincial Key Laboratory of Coronary Heart Disease Prevention, Guangdong Cardiovascular Institute, Guangdong Provincial
People's Hospital, The Affiliated Guangdong Provincial People's Hospital of South China University of Technology, Guangdong Academy of Medical Sciences, Guangzhou, China

${ }^{2}$ School of Pharmacy, Guangdong Pharmaceutical University, Guangzhou, China

${ }^{3}$ Department of Cardiology, Maoming People's Hospital, Maoming, China

${ }^{4}$ Department of Cardiology, First People's Hospital of Kashgar, Kashgar, China

Contributors YL, XL, SX, JL, G-LS, S-QC, XC, XD, YH, FS, YL, Y-YH, YZ, ZC, NT and J-YC: conception and design of the work. $Y L, X L, S X, X C$ and $X D$ : determining search terms, related literature search. YL, XL, SX, JL, G-LS, S-QC, XC, XD, YH and FS: filter literature according to established standards. YL, YH, YZ, ZC, NT and J-YC: as a third author, judge the doubtful literature. YL and S-QC: acquisition, analysis, or interpretation of data. YL, S-QC, J-YC, YL and NT: drafting the work or revising it critically for important intellectual content. All authors agreed that this is the final version of the article. All authors agreed to be accountable for all aspects of the work in ensuring that questions related to the accuracy or integrity of any part of the work are appropriately investigated and resolved.

Funding The study is supported by the Science and Technology Planning Project of Guangdong Province (grant no. 2014B070706010), The Technology Planning Project of Dongguan Province (grant no. 2015108101022), The National Science Foundation for Young Scientists of China (grant no. 81500520), and The Progress in Science and Technology Project of Guangdong Province (grant no. 2015A030302037).

Competing interests None declared.

Patient consent for publication Not required.

Provenance and peer review Not commissioned; externally peer reviewed.

Open access This is an open access article distributed in accordance with the Creative Commons Attribution Non Commercial (CC BY-NC 4.0) license, which permits others to distribute, remix, adapt, build upon this work non-commercially, and license their derivative works on different terms, provided the original work is properly cited, appropriate credit is given, any changes made indicated, and the use is non-commercial. See: http://creativecommons.org/licenses/by-nc/4.0/.

\section{REFERENCES}

1. Tsai TT, Patel UD, Chang TI, et al. Contemporary incidence, predictors, and outcomes of acute kidney injury in patients undergoing percutaneous coronary interventions: insights from the NCDR Cath-PCI registry. JACC Cardiovasc Interv 2014;7:1-9.

2. James MT, Samuel SM, Manning MA, et al. Contrast-Induced acute kidney injury and risk of adverse clinical outcomes after coronary angiography: a systematic review and meta-analysis. Circ Cardiovasc Interv 2013:6:37-43.

3. Mehran R, Dangas GD, Weisbord SD. Contrast-Associated acute kidney injury. N Engl J Med 2019;380:2146-55.

4. Gurm HS, Seth M, Kooiman J, et al. A novel tool for reliable and accurate prediction of renal complications in patients undergoing percutaneous coronary intervention. J Am Coll Cardiol 2013;61:2242-8.

5. Mehran R, Aymong ED, Nikolsky E, et al. A simple risk score for prediction of contrast-induced nephropathy after percutaneous coronary intervention: development and initial validation. J Am Coll Cardiol 2004;44:1393-9.

6. Allen DW, Ma B, Leung KC, et al. Risk prediction models for contrastinduced acute kidney injury accompanying cardiac catheterization: systematic review and meta-analysis. Can J Cardiol 2017;33:724-36.

7. Silver SA, Shah PM, Chertow GM, et al. Risk prediction models for contrast induced nephropathy: systematic review. BMJ 2015;351.

8. Wells GA, Shea B, O'Connell D, et al. The Newcastle-Ottawa Scale (NOS) for assessing the quality of nonrandomised studies in metaanalyses [webpage on the Internet. Ottawa, ON: Ottawa Hospital Research Institute, 2011.

9. Sterne JAC, Hernán MA, Reeves BC, et al. ROBINS-I: a tool for assessing risk of bias in non-randomised studies of interventions. BMJ 2016;355.

10. Shamseer L, Moher D, Clarke M, et al. Preferred reporting items for systematic review and meta-analysis protocols (PRISMA-P) 2015: elaboration and explanation. BMJ 2015;350:g7647. 